\title{
Quantum tunneling fragmentation model
}

\author{
Borko D. Stošić* and M. A. F. Gomes ${ }^{\dagger}$ \\ Departamento de Física, Universidade Federal de Pernambuco, 50670-901 Recife-PE, Brazil \\ Sadhan K. Adhikari \\ Instituto de Física Teórica, Universidade Estadual Paulista, 01405-900 São Paulo, SP, Brazil
}

(Received 12 August 1999; revised manuscript received 3 January 2000)

\begin{abstract}
A nonthermal quantum mechanical statistical fragmentation model based on tunneling of particles through potential barriers is studied in compact two- and three-dimensional systems. It is shown that this fragmentation dynamics gives origin to several static and dynamic scaling relations. The critical exponents are found and compared with those obtained in classical statistical models of fragmentation of general interest, in particular with thermal fragmentation involving classical processes over potential barriers. Besides its general theoretical interest, the fragmentation dynamics discussed here is complementary to classical fragmentation dynamics of interest in chemical kinetics and can be useful in the study of a number of other dynamic processes such as nuclear fragmentation.
\end{abstract}

PACS number(s): 05.40.-a, 64.60.Ht, 73.40.Gk, 25.70.Mn

\section{INTRODUCTION}

Fragmentation is one of the most studied physical phenomena, with applications ranging from crushing [1] and grinding [2] of solids to droplet breakup [3], and disassembly of heavy nuclear [4] or large molecular [5] structures in highenergy collisions, among many others [6]. Usually, experiments in fragmentation are modeled by simple geometric algorithms [7] or by phenomenological approaches [8,9] in order to describe the statistical features of the processes. Only classical concepts are used in these descriptions and in general, quantum processes have not been explicitly considered. As a further example of a classical fragmentation dynamics we have the pioneering numerical simulations reported in Refs. [10] and [11], which consider reactive processes in the kinetic and diffusion-limited regimes on a porous media. Along these classical lines, we have introduced in a previous publication [12] a fragmentation model of interest in chemical kinetics and related phenomena, e.g., the attack on a piece of metal by corrosive rain, in which the reactivity or probability $p_{i}$ of a successful attack at a lattice site $i$ is given by the Boltzmann factor:

$$
p_{i}=\exp \left(-E_{i} / k_{B} T\right)
$$

with $E_{i}=q_{i} E, q_{i}$ the coordination number of site $i, E$ the characteristic energy of each of the $q_{i}$ chemical bonds, $k_{B}$ the Boltzmann constant, and $T$ the temperature. At each time step, a site $i$ on the lattice is chosen at random. The atom or molecule at that site changes its chemical status, and is eliminated off the lattice (i.e., it diffuses from the lattice) with probability $p_{i}$. Obviously, the continued action of this dy-

\footnotetext{
*On leave from Laboratory for Theoretical Physics, Institute for Nuclear Sciences Vinča, P. O. Box 522, YU-11001 Belgrade, Yugoslavia. Electronic address:borko@npd.ufpe.br

${ }^{\dagger}$ Electronic address: mafg@npd.ufpe.br

‡Electronic address: adhikari@ift.unesp.br
}

namics leads to the fragmentation of the system. In this classical fragmentation dynamics, the thermal fluctuations control the likelihood that the chemical bonds will break and as a consequence they also control the evolution of the fragmentation pattern.

In the present paper we are, on the contrary, interested in the study of a fragmentation model controlled by quantum fluctuations at zero temperature. In this model the dynamics obeys different statistics, with quantum tunneling of particles through potential barriers replacing the classical transition probability of Eq. (1). Tunneling is one of the most important consequences of the wave properties of matter and had its first successful application in nuclear fragmentation phenomena such as $\alpha$ decay $[13,14]$ and spontaneous fission, in general. Besides its general theoretical interest, the fragmentation dynamics discussed here is complementary to the classical fragmentation dynamics based on Eq. (1), and perhaps may be useful in the study of a number of other dynamical processes such as fragmentation of heavy nuclei in high energy collisions.

In Sec. II, we describe the quantum tunneling fragmentation model and give details of our Monte Carlo simulations with this dynamics. In Sec. III, we present the numerical results and make a comparison with other fragmentation dynamics. Our major conclusions are summarized in Sec. IV.

\section{QUANTUM-TUNNELING FRAGMENTATION MODEL}

\section{A. Description of the model}

The quantum tunneling fragmentation (QTF) model studied here describes the decay and fragmentation of a large system via tunneling of particles of mass $m$ and energy $E$ through potential barriers of a characteristic height $V_{0}>E$ and variable length $L_{i}$. In this case, the usual quantum mechanical probability for tunneling through the barrier is controlled by the factor [15]

$$
p_{i}=e^{-L_{i} / L_{0}}, L_{0}=\hbar / 2 \sqrt{2 m\left(V_{0}-E\right)} .
$$




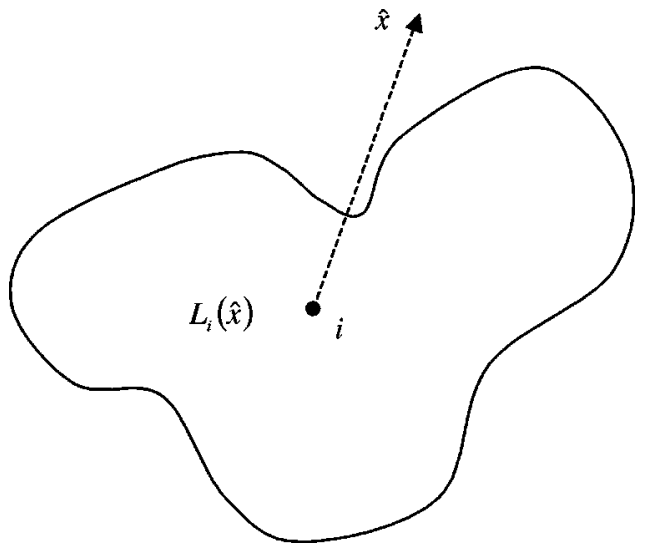

FIG. 1. Ilustrates the distance $L_{i}=L_{i}(\hat{x})$ used in Eq. (2): $L_{i}$ is the shortest distance from site $i$ to the boundary of the system.

The QTF model obeys the following rules:

(i) A site $i$ belonging to a $d$-dimensional square lattice of size (initial mass) $L^{d}$ is chosen at random at each time step.

(ii) If site $i$ is unoccupied, it remains so. If it is occupied, it is unoccupied with the probability given by Eq. (2). The procedure is repeated until all sites are vacant. More precisely, the tunneling explained at the beginning of this paragraph is performed along the direction $\hat{x}$ that minimizes the distance $L_{i}=L_{i}(\hat{x})$ from the site $i$ to the system's boundary as illustrated in Fig. 1, in order to maximize the tunneling probability (2).

Thus while in the classical simulations of Ref. [12], the reactivity or probability of a successful attack depends only on the local variable $E_{i}=q_{i} E$, in the QTF model, $p_{i}$ as given by Eq. (2) is a function of the global variable $L_{i}(\hat{x})$. This variable depends on both the site $i$ and the boundary of the system/fragment and evolves continuously, changing with the connectivity of the system and its fragmentation. After each tunneling the system is reduced by one unit of mass.

In principle, two types of different physical situations can be associated with rule (ii) of the QTF model. First, we could have a direct process in which the tunneling particle is a quantum particle at site $i$. It tunnels from $i$, leaving the site $i$ unoccupied and reducing the mass of the system by one unit. Secondly, we have an indirect process in which we could assume, in the spirit of Born-Oppenheimer approximation [15], that the tunneling particle is some kind of lighter particle that bounds heavier clusters (of unit mass) localized at $i$ to the entire system. If the lighter particle tunnels, the heavy particle is no longer bound to the system and it is free to go away reducing the mass of the system by one unit. Finally, we note in passing that the physical processes underlying the QTF model are possible in any space dimension $d$, contrarily to the classical model of Ref. [12], which is more physical in one and two space dimensions.

\section{B. Simulation}

The simulation itself is conceptually simple: lattice sites are visited randomly, and removed with probability $p_{i}$ given by Eq. (2). As described above, the probability of successful attack $p_{i}$, on the particle at site $i$, depends on its distance from boundary at the moment of attack. As any such event subsequently places all the occupied neighboring sites on the boundary, the boundary itself dynamically changes. The probability of tunneling therefore depends on a global, dynamically changing variable. The computational effort needed for such an algorithm dramatically increases as compared to the local models, since for every lattice site one needs to check (in principle) all the other sites in order to determine the closest boundary point.

To optimize the use of the available hardware resources, we have developed an algorithm using linked lists, to keep track of the boundary sites. More precisely, information as to which sites currently belong to the boundary is kept separately, in the form of a list of elements that point to each other. The first boundary element points to the second, the second to the third, and so on until the last, which points to the first. Starting from any of the boundary sites one cycles through the whole boundary without the need of visiting the non-boundary sites. When a new site needs to be added to the boundary, the chain is broken (at an arbitrary position), and reconnected to include the new site. For example, to add a site $C$ between sites $A$ and $B$, where $A$ was pointing to $B$, one simply makes $A$ point to $C$ and $C$ to $B$, leaving $B$ unchanged. Similarly, to remove $C$ from the $A C B$ list, one makes $A$ point to $B$, while the pointer of $C$ is set to zero.

Determining the fragment size distribution along the simulation poses another challenge from the computational point of view, which is, however, common with the classical fragmentation models. We have used the Hoshen-Kopelman algorithm developed for numerical studies of percolation phenomena [16,17], which appears to be the most efficient of such algorithms developed up to date.

The simulation dynamics is rather sensitive to the quality of the random number generator. The generators which produced notably different results from each other failed to pass the Marsaglia's Diehard battery of tests [18]. Our final choice is the combined multiply-with-carry generator with period exceeding $2^{60}$.

Another important point turns out to be the choice of the characteristic length scale $L_{0}$ in Eq. (2). If it is taken to be too small [i.e., if $\left(V_{0}-E\right)$ is large], the fragmentation process is confined to the surface of the sample, with a single large cluster. On the other hand, if $L_{0}$ is very large $\left[\left(V_{0}\right.\right.$ $-E$ ) small], fragmentation takes place throughout the sample volume, resembling the classical thermal model of Ref. [12] at infinite temperature. The situation that most deviates from the classical models is obtained when $L_{0}$ is taken to be considerably smaller than the sample size $L$, but not too small.

In the present study on fragmentation we consider the variable diversity of fragments $D(t)$, which gives the number of different types of fragments. The diversity of fragments $D(t)$ is defined by

$$
D(t)=\sum_{s} \theta[n(s, t)]
$$

where $n(s, t)$ is the distribution function of fragments of mass $s$ at time $t$, and $\theta(x)$ is the step-function satisfying $\theta(x)=1$, for $x>0$, and $\theta(x)=0$, for $x \leqslant 0$. The evolution of diversity with time in several situations is shown in Fig. 2. Data are shown for a simulation performed on a $512 \times 512$ 


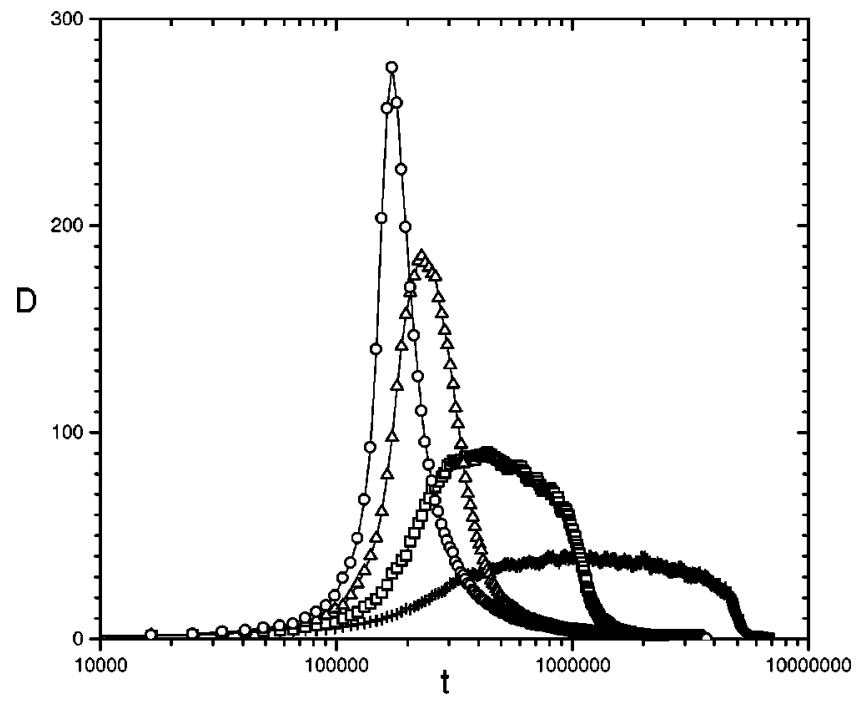

FIG. 2. Diversity of fragments $D(t)$ as a function of time, for characteristic length choices $L_{0}=1(+), L_{0}=2(\square), L_{0}=4(\Delta)$, and $L_{0}=8(\bigcirc)$. These results refer to QTF model simulations on square lattices with $512^{2}$ sites, and the statistical functions are averages over eight samples.

system in two dimensions, for $L_{0}=1(+), L_{0}=2(\square), L_{0}$ $=4(\Delta)$, and $L_{0}=8(\bigcirc)$. It is seen that the curves for $L_{0}$ $=1$ and $L_{0}=2$ exhibit a plateau-type broad maxima rather than a clear pronounced maximum in diversity. This is due to the fact that when $L_{0}$ is small in comparison with $L$ the fragmentation process is confined to a narrow region around the surface, while the bulk is practically unaffected by the fragmentation dynamics. This region is formed on the very beginning of the simulation (corresponding to the initial rise of diversity), after which it drifts at constant rate towards the interior (corresponding to the plateau). Finally, when the opposite sides of the affected region meet at the center of the system, the diversity rapidly decays. Increasing the characteristic length $L_{0}$ increases the width of the affected region, yielding a sharp diversity peak. On the other hand, if $L_{0}$ is taken to be too large (in comparison with $L$ ), the whole system is immediately exposed to fragmentation dynamics, and distance of a certain point from the boundary ceases to be a relevant parameter. After extensive initial tests on systems of various sizes in both two and three dimensions, we have opted for $L_{0}=L / 64$, which in all cases yields a reasonably sharp diversity maximum, and is on the other hand sufficiently removed from the classical model.

Simulations were performed on a 64-bit UltraSparc HPC 3000 SUN station, investing several hundred hours of CPU time. For each of the studies, 16 to 32 simulations were performed independently to reduce statistical fluctuations. Finally, an averaging was taken over these independent simulations. The largest sample sizes that we were able to study are $1024 \times 1024$ in two dimensions, and $64 \times 64 \times 64$ in the three-dimensional case.

\section{RESULTS AND DISCUSSION}

Most experiments in fragmentation examine mainly the mass (size) distribution of fragments, $n(s)$, which gives the number of fragments of mass (size) $s$ after the application of

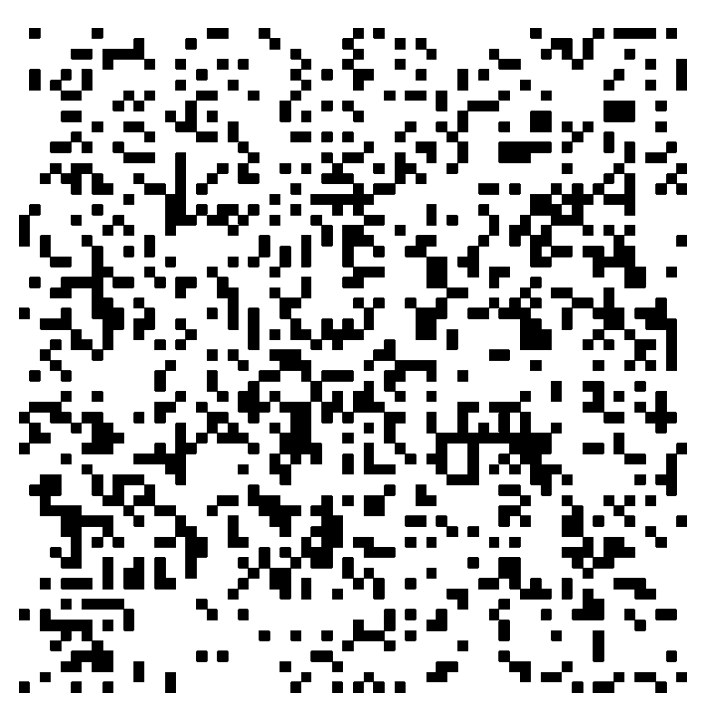

FIG. 3. Typical distribution of fragments in a single realization of the $2 d$ QTF model at the time of maximum diversity of sizes for a system of initial mass $64^{2}$.

an impulsive force, after an explosion or collision, or after some other particular process of breakup [8,19-21]. From the practical point of view, the cumulative distribution of fragments $N(s)$, which is the number of fragments with mass greater than $s$, is first obtained and then the differential mass distribution $n(s)$ is found. If $N(s) \sim s^{-\kappa}$, we have $n(s)$ $\sim s^{-\tau}$, with $\tau=\kappa+1$. However, these scaling relations are not much informative because, in spite of the nonuniversality of $\tau$, it can be shown under very general conditions $[8,9]$ that many classes of completely different fragmentation dynamics lead to only a small variation of $\tau$ (typically $1.7 \leqslant \tau$ $\leqslant 2$ ). To avoid this insensitivity to dynamics, other statistical quantities should be investigated in fragmentation experiments, but this is not a simple matter. Recently, we made an effort in this direction by studying the statistics of the diversity of fragments in a simple fragmentation experiment [22].

In the present paper, we report the results of a detailed statistical study of the QTF model in two and three dimensions. The differential distribution of fragments, $n(s)$, as well as the diversity of fragments, $D(t)$, the total mass, $M(t)$, and the total number of fragments, $N(t)$, and other statistical functions, such as the mass concentrated at the boundaries of the system, $B(t)$, as a function of the time are also investigated.

\section{A. QTF model in $d=2$}

In order for the reader to develop some insight about the QTF model, we show in Fig. 3 a typical distribution of fragments obtained in a single experiment, at the time of maximum diversity of sizes of fragments, for a simulation with a system of initial mass $64^{2}$.

In Fig. 4 we show the total mass, $M(t)(\Delta)$, the mass on the boundary or total perimeter, $B(t)(\nabla)$, which is defined as the number of occupied sites with less than four nearestneighbors, the total number of fragments, $N(t)(\bigcirc)$, and the diversity of fragments, $D(t)(\square)$, defined by Eq. (3). We can see from these plots

$$
B(t) \sim t^{\beta}, \quad \beta=0.95 \pm 0.05, \quad 0.01 \leqslant t / t_{D_{\max }} \leqslant 0.28
$$




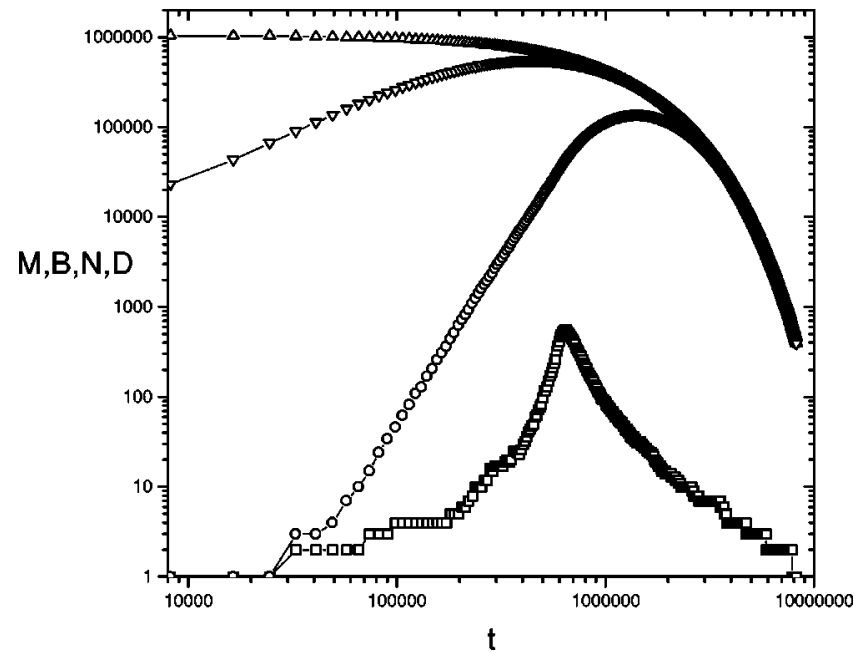

FIG. 4. The total mass, $M(t)(\Delta)$; the mass on the boundary, $B(t)(\nabla)$; the total number of fragments, $N(t)(\bigcirc)$; and the diversity of fragments, $D(t)(\square)$, as a function of the time, for the QTF model in $d=2$. These results refer to simulations on square lattices with $1024^{2}$ sites, and the statistical functions are averages on 16 samples.

where $t_{D_{\max }}$ is the time where $D(t)$ is maximum, i.e., the time at which there exists the largest number of length or mass scales. The total number of fragments, on the other hand, satisfies

$$
N(t) \sim t^{\nu}, \quad \nu=3.7 \pm 0.2, \quad 0.07 \leqslant t / t_{D_{\max }} \leqslant 1
$$

i.e., the dynamic scalings in $B(t)$ and $N(t)$ coexist in the interval $0.07 \leqslant t / t_{D_{\max }} \leqslant 0.28$ in which the average fragment size $\langle s(t)\rangle=M(t) / N(t)$ behaves as

$$
\langle s(t)\rangle \sim t^{-z}, \quad z=3.9,
$$

as indicated in Fig. 5. It can be noticed that the scaling in $\langle s(t)\rangle$ extends over almost five decades in $\langle s\rangle$. The reader

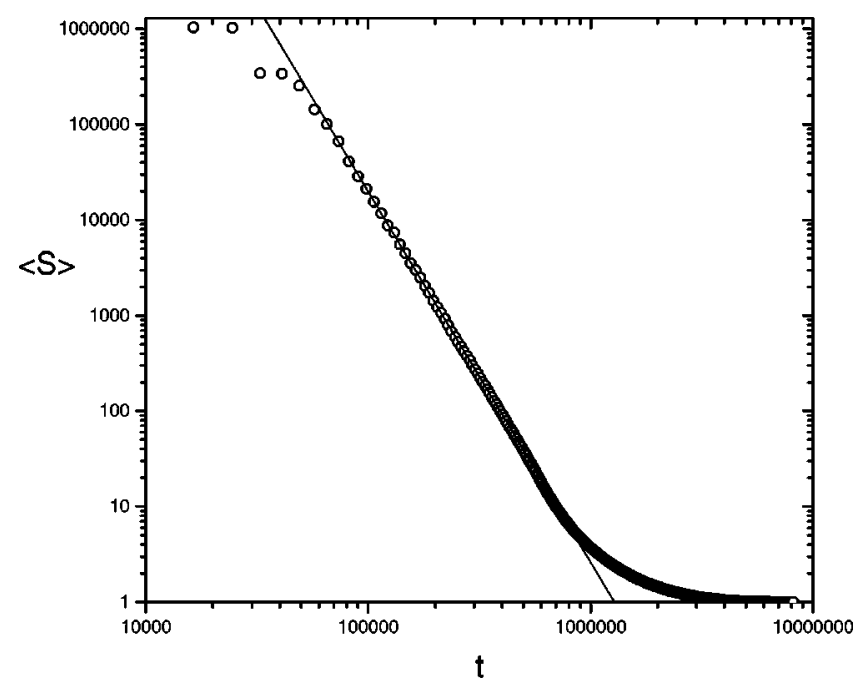

FIG. 5. The time dependence of the average fragment size, $\langle s\rangle$, for the simulations of Fig. $4 .\langle s\rangle$ scales as $t^{-z}, 0.08 \leqslant t / t_{D_{\max }} \leqslant 1$ (see Sec. III A for details). The straight line has the slope $-z=-3.9$ \pm 0.2 . can notice that from Eqs. (5) and (6) we get $d M(t) / d t$ $=d[N(t)\langle s(t)\rangle] / d t \sim(\nu-z) t^{\nu-1-z}$. However, as can be seen from Fig. 4, the scalings in time for $N(t)$ and $\langle s(t)\rangle$ are valid in an interval where $d \ln M(t) / d \ln t=[t / M(t)] d M(t) / d t$ is quite small and, as a consequence, $\nu \approx z$, in a first approximation. On the other hand, the total perimeter $B(t) \sim t^{\beta}[\mathrm{Eq}$. (4)] can also be expressed as $B(t) \sim N(t)\langle s(t)\rangle^{\alpha} \sim t^{\nu-\alpha z}$, where $\langle s(t)\rangle^{\alpha}$ is the perimeter for the average fragment, and $\alpha<1$. Equations (4) $-(6)$ leading to $\beta=\nu-\alpha z$ are compatible with a perimeter exponent $\alpha=0.7 \pm 0.1$, a result that is somewhat larger than the Euclidean value $\alpha=(d-1) / d$ $=1 / 2$, for $d=2$.

As the variable diversity of fragments plays an important role in fragmentation processes, it seems opportune to comment here on its physical meaning. The concept of diversity has been used in an increasing number of scientific works in connection with biological [23] and volutionary [24] problems, as well as in relation to self-organization, cellular automata [25], and fractals [26], among others. In fluid mechanics, and in many other physical phenomena, complex behavior is associated with a spatial inhomogeneity, i.e., with a diversity in size scales [27]. In the last few years, an effort has been made to classify complex configurations which often arise from simple algorithms, as well as to define measures of complexity [28]. Recently, we have proposed that the diversity of size of fragments defined in Eq. (3) is a good variable to measure the complexity in fragmentation experiments [29]. Despite its intrinsic and technological relevance, the diversity of size in fragmentation processes is a relatively unknown subject since it cannot be easily derived from the equations underlying the dynamics. In fragmentation, the diversity [defined by Eq. (3)] first increases, then attains its maximum when the system assumes the most complex configuration, and later it decreases again [29]. Computer simulations for several fragmentation dynamics have shown that in the space of lower (higher) dimension a large diversity is easily (hardly) generated, however, it is also quickly (slowly) destroyed [29]. From the experimental point of view, only recently the diversity of size has been examined for the first time in a fragmentation experiment of brittle solids under the application of a repeated impulsive force [22], and several (scaling) relations involving this variable were observed, in agreement with previous results obtained with numerical simulations.

Although $D(t)$ given by (3) does not present dynamic scaling, there are interesting relationships involving the maximum $D_{\max }$ of this quantity. First of all, it can be noticed from Fig. 4 that the maxima of $B(t)$ and $D(t)$ occur near the same time $t_{D_{\max }}$. This particular time, in which the number of length or mass scales is maximum depends on the lattice size $L$ as

$$
t_{D_{\max }} \sim L^{1.5}
$$

as shown in Fig. 6 for $32 \leqslant L \leqslant 1024$. Thus, as the size of the system increases, the system spends proportionally more time to attain the state of largest diversity of length scales. Furthermore, $D_{\max }$ obeys the following scalings:

$$
D_{\max } \sim B_{\max }^{\delta}
$$




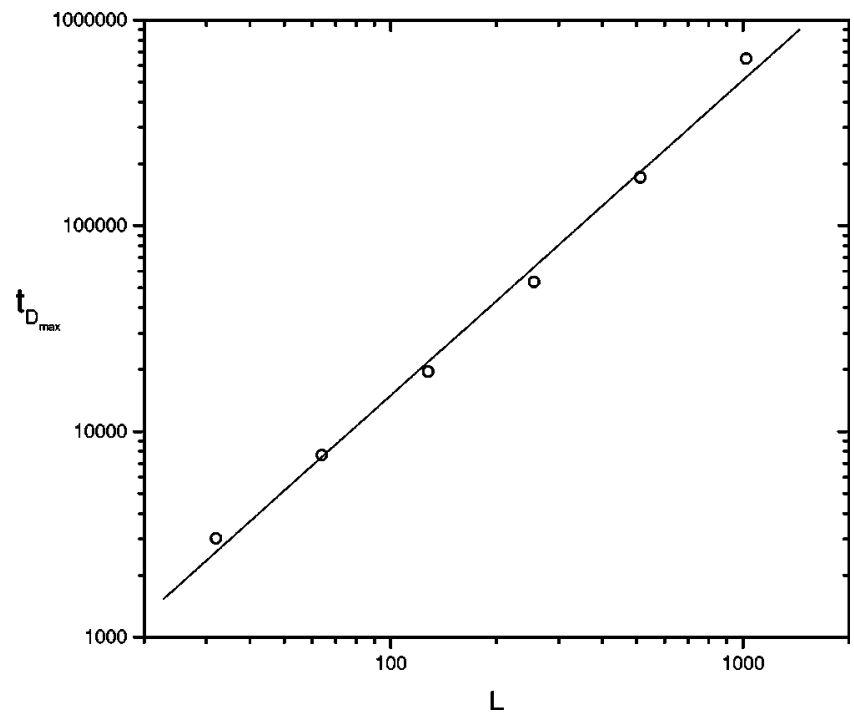

FIG. 6. The time needed to reach the maximum diversity of sizes, $t_{D_{\max }}$, as a function of the size $L$ of the lattice. The straight line represents the best fit $t_{D_{\max }} \sim L^{1.5 \pm 0.1}(32 \leqslant L \leqslant 1024)$ to the data.

$$
D_{\max } \sim N_{\max }^{\delta}
$$

with $\delta=0.5 \pm 0.05$, as indicated in Figs. 7 and 8, respectively. $N_{\max }$ must be an extensive variable in the thermodynamic limit, thus $N_{\max } \sim L^{2}$ and, as a consequence, $D_{\max }$ $\sim B_{\max }^{1 / 2} \sim L$.

The distribution function $n(s)$ of fragments of mass $s$ at time of maximum diversity for a system of initial mass $1024^{2}$ is shown in Fig. 9. The data in this figure correspond to an average over 16 equivalent experiments. As we can see in Fig. 9, the simulation data lead to a power law along two decades in $s$, with

$$
n(s) \sim s^{-\tau}, \quad \tau=1.7 \pm 0.1
$$

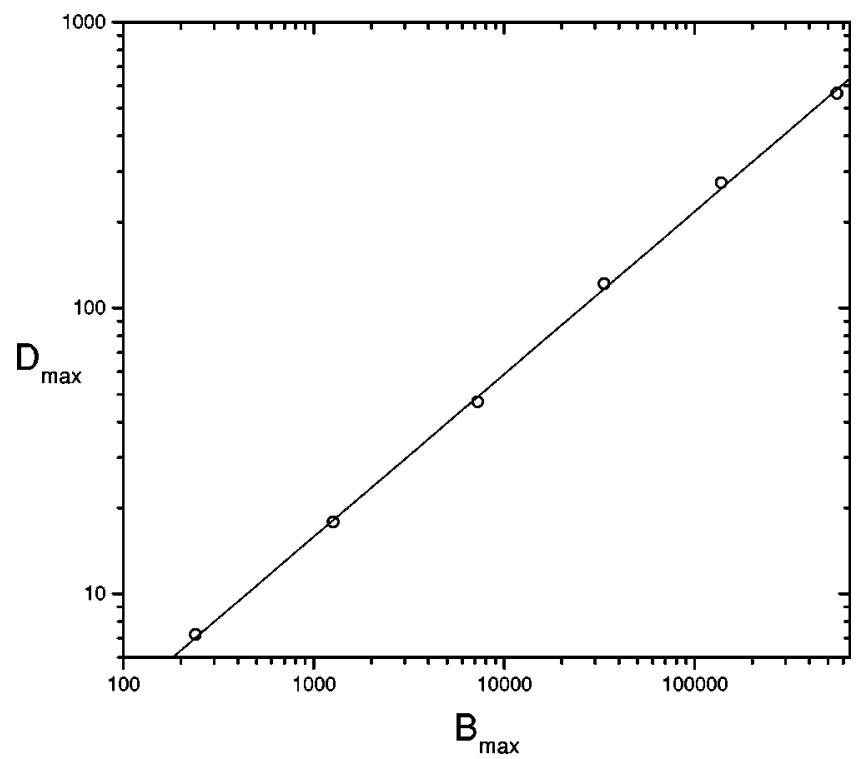

FIG. 7. The maximum diversity of sizes, $D_{\max }$, scales with the maximum mass on the boundary, $B_{\max }$, as $D_{\max } \sim B_{\max }^{1 / 2}$, for $32 \leqslant L$ $\leqslant 1024$. The straight line has a slope of $1 / 2$ with a $95 \%$ confidence limit.

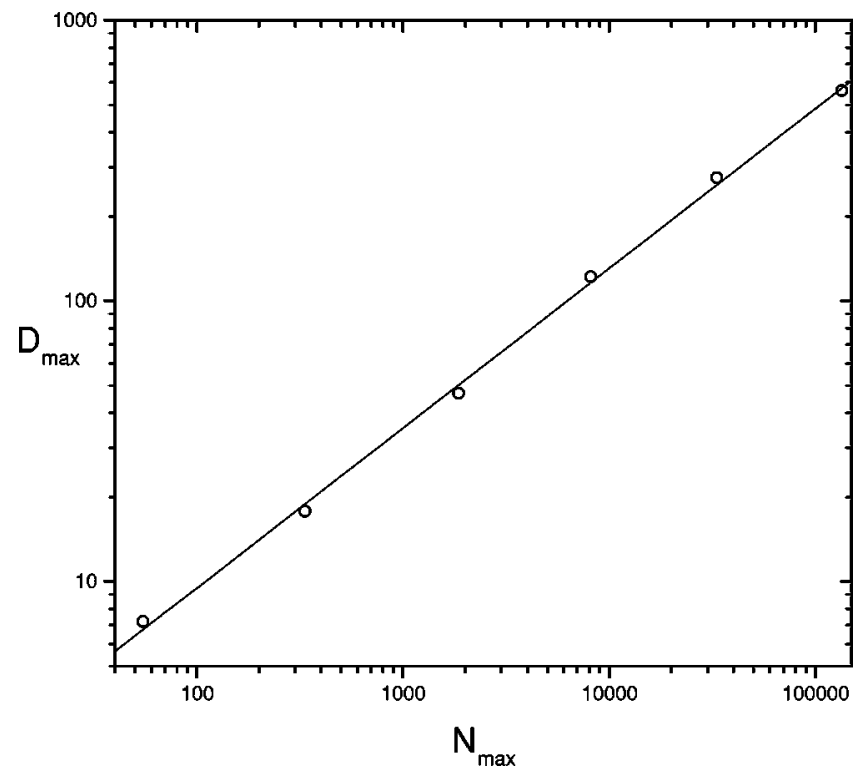

FIG. 8. The dependence of the maximum diversity, $D_{\max }$, with the maximum of the total number of fragments, $N_{\max }$, for $32 \leqslant L$ $\leqslant 1024$. The straight line represents the best fit to the data and has a slope of $1 / 2$ with a $95 \%$ confidence limit. See Sec. III A for details.

For the sake of completeness it is interesting to remark that the value of the exponent $\tau$ obtained in the QTF model [Eq. (10)] is in the same universality class of a number of dynamical problems that seem completely unrelated, e.g., the fragmentation of brittle platelike objects $[22,30]$, the distribution of undissolved metal islands in pitting corrosion [31], and the fragmentation of invasion percolation like structures in $2 \mathrm{~d}$ fluids [32], among others.

To illustrate the differences among the critical exponent $w$ for different values of mass, in Fig. 10 we exhibit the time dependence of $n(s, t)$ for $s=1,2$, and 4 . From this figure, we obtain the scaling

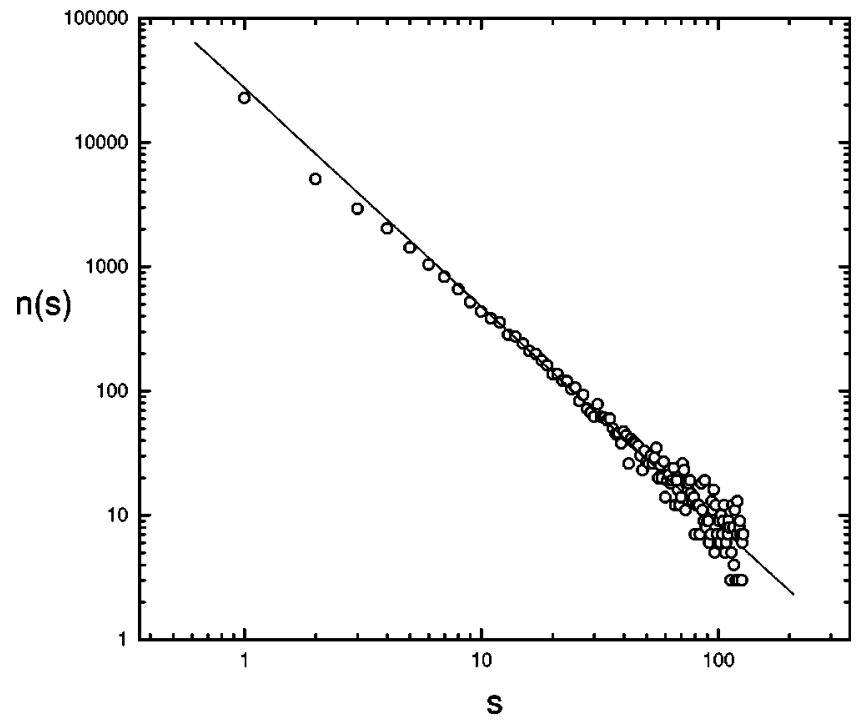

FIG. 9. The differential distribution of fragments of size $s, n(s)$, at the time of maximum diversity, for the QTF model in $d=2$ on a system of initial mass $1024^{2}$. The straight line gives the best fit to the data: $n \sim s^{-\tau}, \tau=1.7 \pm 0.1$. See Sec. III A for details. 


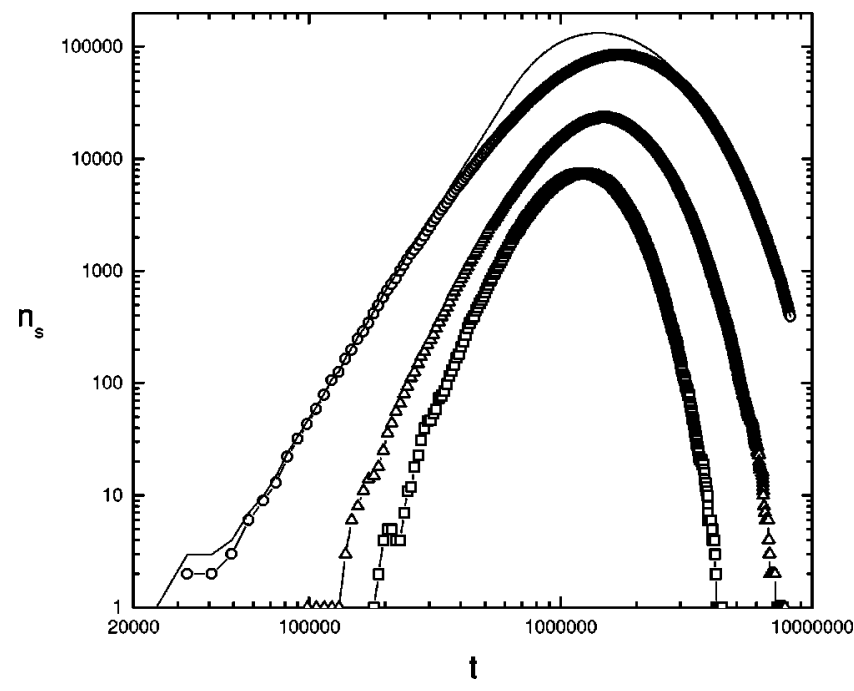

FIG. 10. The time dependence of the distribution of fragments $n(s, t)$ for fragments of size $s=1(\bigcirc), 2(\Delta)$, and $4(\square)$, obtained from simulation on a lattice of $1024^{2}$ sites, avaraged over 16 samples. The continuous line represents the integral function $N(t)$. Each size $s$ is associated with a different dynamic exponent $w_{s}$ as explained in Sec. III A (Eq. 11).

$$
n_{s}(t) \sim t^{w_{s}}, \text { with } w_{s}=w s^{\gamma}, \quad w=3.7, \quad \gamma=0.30
$$

Equation (11) shows that a single exponent $w$ cannot account for the dynamic evolution of the entire distribution of fragments in the scaling region. A sequence of exponents $w_{s}$, one for each size $s$ (an infinite family of exponents in the thermodynamic limit $L \rightarrow \infty$ ), is needed as, e.g., in multifractal [33] and multiscaling [34] phenomena in statistical physics.

It is interesting to observe that scaling (11) is essentially the same result obtained with the classical fragmentation dynamics of Ref. [12], where the probability of successful attacks is controlled by the Boltzmann factor as in Eq. (1). Also, the exponents $\tau$ in Eq. (10) and $\nu$ in Eq. (5) are the same exponents reported in Ref. [12]. The exponent $z=3.9$ of Eq. (6) is somewhat larger than the classical exponent $z$ $=3.5$ of Ref. [12]. Thus, among the four important exponents describing the scaling laws in fragmentation dynamics $(\nu, z, \tau$, and $w)$, only the dynamic exponent $z$ presents a small deviation from the classical values.

The fragmentation dynamics studied in the present article and in Ref. [12] have in common the fact that the defects, or the attack that lead to the fragmentation of the original matrix are uncorrelated point defects, i.e., they have topological dimension $d_{T}=0$. Other fragmentation dynamics [35,36], using extended (diffusive) defects as random walks (i.e., sequences of highly correlated point defects with $d_{T}=1$ ) give quite different values for the exponents $\nu, z$, and $w$, when compared with the case $d_{T}=0$. Physically, the two classes of fragmentation dynamics are quite different. In the first case $\left(d_{T}=0\right)$, it is the coalescence of a number of uncorrelated point-defects, e.g., the uncorrelated voids or single broken chemical bond, that generate new closed interfaces enclosing fragments. In the second case $\left(d_{T}=1\right)$ it is the coalescence of one-dimensional objects, i.e., highly correlated strings of contiguous points, that generate the new closed interfaces leading to fragmentation.

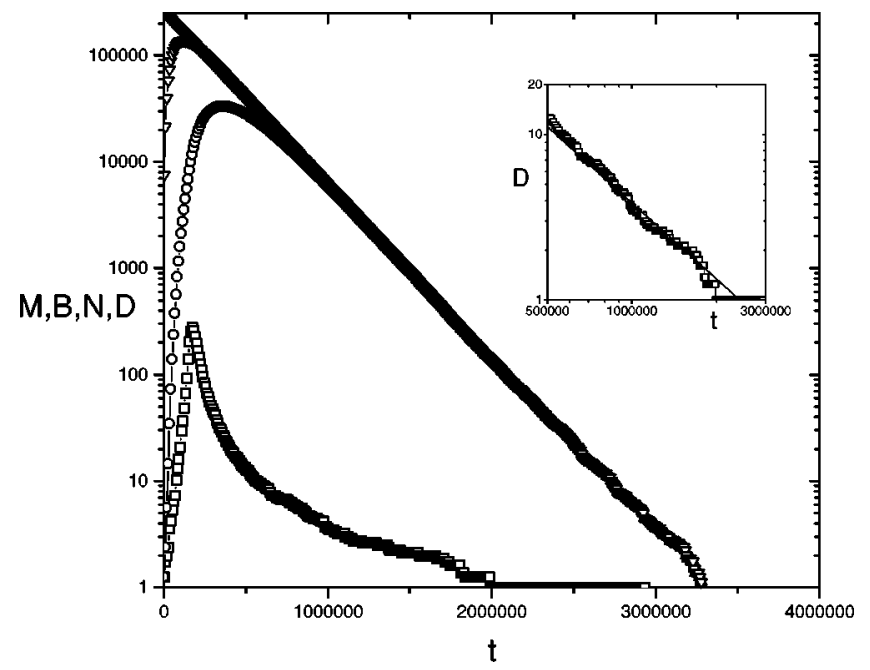

FIG. 11. The same data as in Fig. 4, shown on a semilogarithmic scale. $M(t)(\Delta), B(t)(\nabla)$, and $N(t)(\bigcirc)$, clearly exhibit exponential decay at large times, while $D(t)(\square)$, shows power law behavior, as shown in the inset.

The exponent $\tau$ exhibits a small variation in the cases $d_{T}=0$ and $d_{T}=1$. In both cases, $\tau$ lies in the narrow interval $1.6 \leqslant \tau \leqslant 2.1$. A detailed examination of the simulation data of the present article and also of Refs. [12,35,36] seems to indicate that for $d=2, \nu=3.7$, for $d_{T}=0$, and $\nu=1.1$, for $d_{T}=1$, irrespective the dynamics. Furthermore, $z$ fluctuates in the interval $3.5-3.9$ for $d_{T}=0$, but is confined near $z$ $=1.3$, for $d_{T}=1$, irrespective of the dynamics. In fragmentation dynamics with diffusive attacks $\left(d_{T}=1\right)$, one has the exponent $\gamma$ of Eq. (11) equal to zero, i.e., for these dynamics $w_{s}=w=\nu$ is independent of $s$.

Finally, we briefly address the question of the large time behavior of the quantities under study. In Fig. 11 we show $M(t)(\Delta), B(t)(\nabla), N(t)(\bigcirc)$, and $D(t)(\square)$, on the semilogarithmic scale. It is seen that $M(t), B(t)$, and $N(t)$ decay exponentially at large times. In the inset, $D(t)$ is shown on the log-log scale for times for which practically the whole mass of the system is distributed on the boundary, indicating a power-law behavior of diversity at large times.

\section{B. QTF model in $d=3$}

Now, let us turn to the results of the QTF model in the physical $3 d$ space. In Fig. 12 we show $M(t)(\Delta), B(t)(\nabla)$, $N(t)(\bigcirc)$, and $D(t)$. In contrast to the $2 d$ simulations, the scaling in time of $B(t)$ disappears before $B(t)$ attains its maximum value, $B_{\max }$. However, $N(t)$ continues to obey the scaling

$$
N(t) \sim t^{\nu}, \quad \nu=4.0 \pm 0.2, \quad 0.1 \leqslant t / t_{D_{\max }} \leqslant 1 .
$$

The absence of scaling in $B(t)$ for $d=3$ may be due to the existence of an upper critical dimension for the QTF model, with $B(t)$ exhibiting no scaling in time beyond the critical dimension with all critical exponents remaining constant for $d \geqslant 3$. In Fig. 13 we illustrate with a $2 d$ section the effect of the $3 d$ QTF model dynamics on a system with initial mass $64^{3}$. The average fragment size $\langle(t)\rangle$ scales with time as 


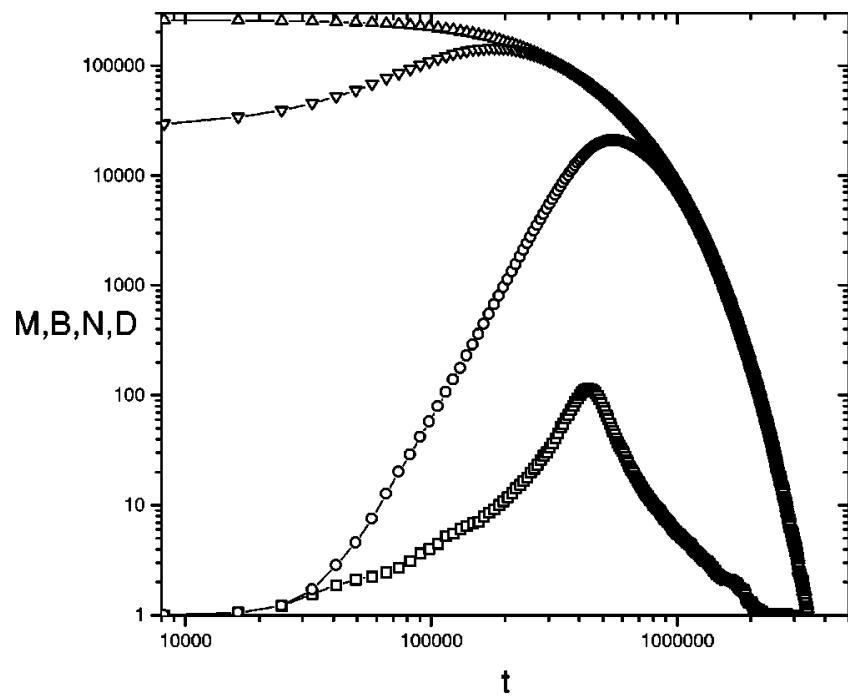

FIG. 12. The same as in Fig. 4 but for the QTF model in $d$ $=3$ on a system of initial mass $64^{3}$. The averages are on 32 samples. See Sec. III B for details.

$$
\langle s(t)\rangle \sim t^{-z}, \quad z=4.5 \pm 0.2, \quad 0.13 \leqslant t / t_{D_{\max }} \leqslant 1
$$

as shown in Fig. 14. Both scalings occur within similar intervals of the variable $t / t_{D_{\max }}$ as in the $2 d$ case.

The distribution $n(s)$ of fragments at the time of maximum diversity for a cubic system of initial mass $64^{3}$ averaged on 32 similar experiments is given in Fig. 15. The distribution $n(s)$ obeys a scaling as in two dimensions [Eq. (10)], with

$$
n(s) \sim s^{-\tau}, \quad \tau=2.1 \pm 0.1
$$

The $3 d$ exponent $\tau=2.1$ is in excellent agreement (within the statistical fluctuations) with the corresponding exponent for $3 d$ percolation [37] $(\tau=2.189)$. The exponent $\tau=2.1$ is somewhat larger than the exponents usually reported in the fragmentation literature $[8,9,20,22,31,32,35]$, an exception

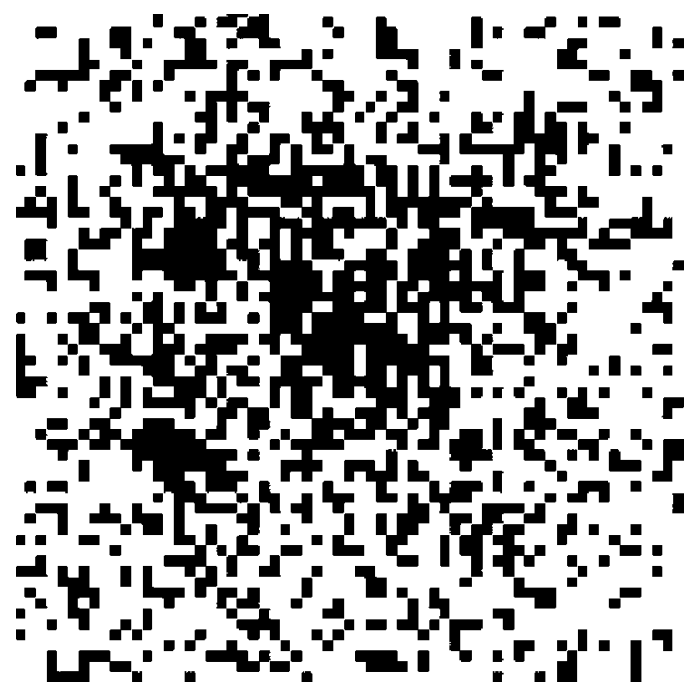

FIG. 13. Typical distribution of fragments in a single realization of the $3 d$ QTF model. The figure refers to a $2 d$ section made at half-height on a system with initial mass $64^{3}$ at the time of maximum diversity of sizes.

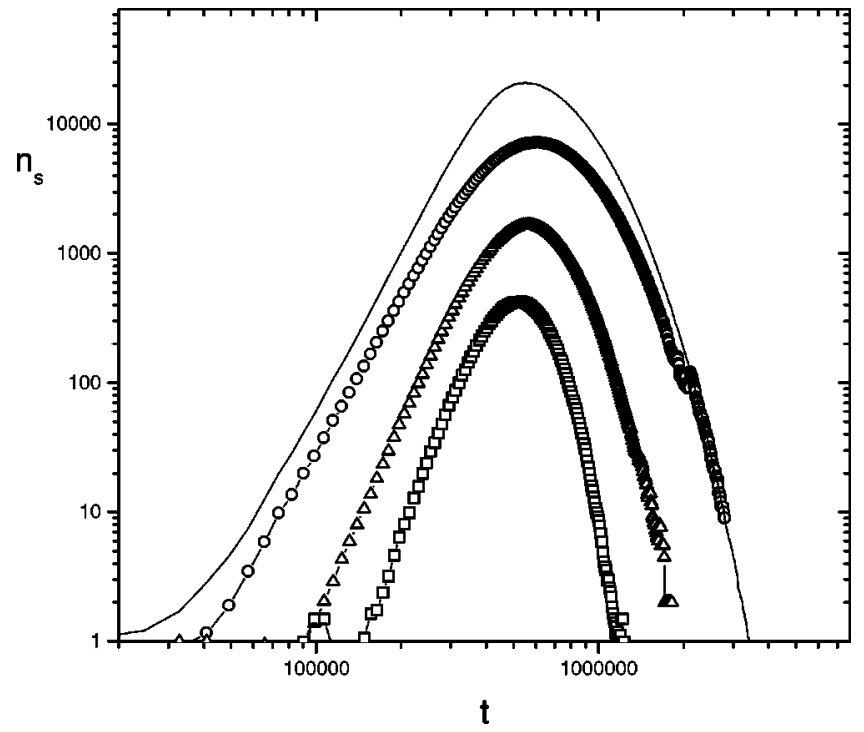

FIG. 14. The same as in Fig. 5 but for the $3 d$ QTF model. The initial mass of the system was $64^{3}$ and the average is on 32 equivalent experiments. The straight line represents the best fit $\langle s\rangle$ $\sim t^{-z}, z=4.5 \pm 0.2$. See Sec. III B for details.

being just the values of the exponent $\tau$ obtained from fragmentation experiments involving collisions of high energetic particles with heavy nuclei $[19,38,39]$. In these nuclear fragmentation experiments, the standard practice is to fit the fragment mass yield with a power law of the type given in Eq. (14) with $2.3 \leqslant \tau \leqslant 2.6$. Obviously, compared to the masses of the systems used in our simulations, nuclear fragmentation uses systems of very small masses (e.g., 209 particles in a typical $\mathrm{C}^{12}+\mathrm{Au}$ high energy collision). Consequently, the value of the exponent $\tau$ and even its existence in nuclear experiments must be taken with caution.

Finally, in Fig. 16 we give the time dependence of the number of fragments of masses $s=1,2$, and $4, n_{s}(t)$ :

$$
n_{s}(t) \sim t^{w_{s}} \text {, with } w_{s}=w s^{\gamma}, w=4.0, \gamma=0.30 \text {, }
$$

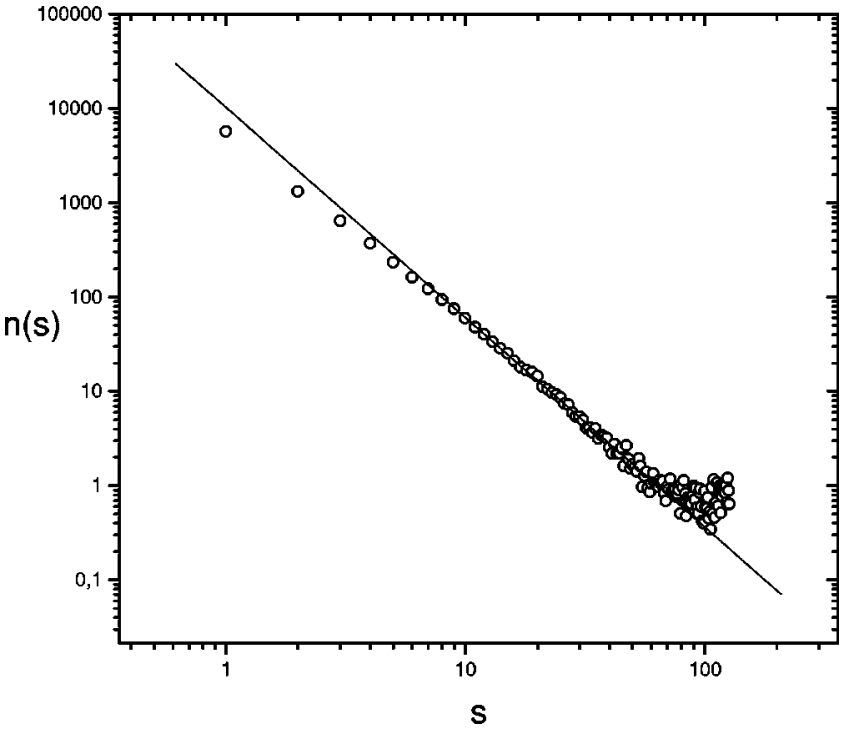

FIG. 15. The same as in Fig. 9 for the $3 d$ QTF model. The straight line represents the best fit to the data: $n \sim s^{-\tau}, \tau=2.1 \pm 0.1$. See Sec. III B for details. 


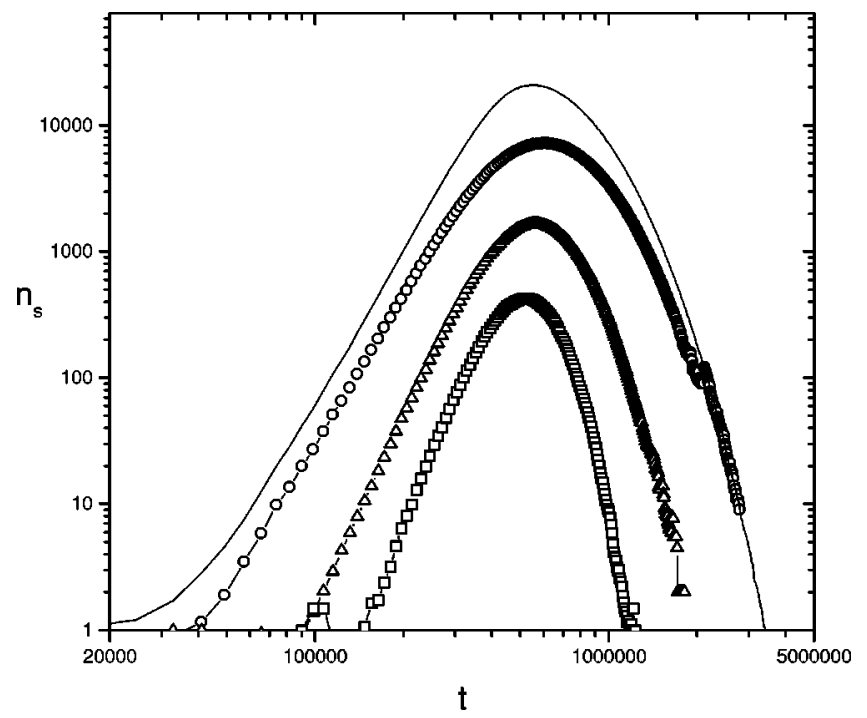

FIG. 16. Time dependence of the distribution function $n(s, t)$, for $s=1(\bigcirc), 2(\Delta)$, and $4(\square)$, obtained from simulations on a lattice of $64^{3}$ sites (averages on 32 samples). The continuous line represents the total number of fragments $N(t)$. See, for comparison, Fig. 10 and Sec. III B for details.

that is, essentially the same distribution for the QTF model in $d=2$ [Eq. (11)], and the classical fragmentation model discussed in Ref. [12]. The exponents $z$ and $\tau$ in $d=3$ [Eqs. (13) and (14)] are somewhat larger than in $d=2$ [Eqs. (6) and (10)]. Furthermore, as observed in $d=2$, the four exponents $\nu, z, \tau$, and $w$ of the QTF model in $d=3$, are essentially the same exponents obtained in the classical fragmentation dynamics of Ref. [12]. For the convenience of the reader, the values of the exponents $\nu, z, \tau, w$, and $\gamma$ shown in Eqs. (5), (6), (10), and (11) are exhibited for $d=2$ and 3 in Table I. These exponents are compared with the corresponding values found in other fragmentation models cited in this section, as well as with the experimental data of Ref. [22].

The QTF model studied in this article makes use of nonthermal processes in compact systems, introducing explicitly quantum tunneling in the fragmentation dynamics. The existence of metastable states separated by a potential barrier from other states is a common feature in physics and it is particularly important in nuclear fragmentation. This fact suggests that the QTF model can be of general interest in nuclear physics to explain the experimental data of mass yields of nuclear fragmentation without resort to equilibrium liquid-gas phase transitions, percolation structures, and other concepts usually considered.
TABLE I. Values of the critical exponents $\nu, z, \tau, w$, and $\gamma$, defined in the scaling relations Eqs. (5), (6), (10), and (11), for the QTF model in dimensions $d=2$ and $d=3$, as well as for other fragmentation models and experimental data cited in Secs. III A and B. The topological dimension $d_{T}$ was introduced in the end of Sec. III A.

\begin{tabular}{lccccccc}
\hline \hline & $d$ & $d_{T}$ & $\nu$ & $z$ & $\tau$ & $w$ & $\gamma$ \\
\hline QTF & 2 & 0 & 3.7 & 3.9 & 1.7 & 3.7 & 0.30 \\
model & 3 & 0 & 4.0 & 4.5 & 2.1 & 4.0 & 0.30 \\
\hline Ref. [12] & 2 & 0 & 3.6 & 3.5 & 1.6 & 3.6 & 0.28 \\
& 3 & 0 & 4.5 & 4.4 & 1.9 & 4.5 & 0.28 \\
\hline Ref. [22] & 2 & 1 & 1.0 & 1.0 & 1.8 & - & - \\
\hline Ref. [35] & 2 & 1 & 0.9 & 1.3 & 1.7 & 0.9 & 0 \\
\hline Ref. [36] & 2 & 1 & 1.2 & 1.2 & - & 1.2 & 0 \\
\hline \hline
\end{tabular}

\section{SUMMARY AND CONCLUSIONS}

We have presented the results of a nonthermal quantum fragmentation dynamics in two- and three-dimensional compact systems making explicit use of tunneling of particles through potential barriers at zero temperature (QTF model). This model is complementary to a classical dynamics previously studied [12] in which particles perform classical transitions over the barrier at finite temperature. These models present several static and dynamic scaling relations, with similar critical exponents in both classical and quantum domain. The time evolution of the total perimeter (area) of the fragments exhibit qualitatively different behaviors in two and three dimensions in the QTF model. The critical exponent $\tau$ in the differential distribution of fragments of mass $s, n(s)$ $\sim s^{-\tau}$, in $d=3$, has the value $\tau=2.1$, which is not significantly different from the exponent obtained in many experiments involving fragmentation of heavy nuclei after high energy collisions $[19,38,39]$. The overall characteristics of the QTF model suggests that it can be relevant in explaining experimental data of nuclear fragmentation as well as in many other fragmentation processes controlled by quantum mechanics, and in many out-of-equilibrium dynamics.

\section{ACKNOWLEDGMENTS}

This work was supported in part by Conselho Nacional de Desenvolvimento Científico e Tecnológico, Financiadora de Estudos e Projetos, Fundação de Apoio à Pesquisa do Estado de São Paulo, and Coordenação de Apoio aos Professores do Ensino Superior (Brazilian Agencies).
[1] P. Rosin and E. Rammler, J. Inst. Fuel 7, 29 (1933).

[2] L.G. Austin, P.T. Luckie, and R.R. Klimpel, Trans. Soc. Min. Eng. AIME 252, 87 (1972).

[3] O.S. Costa, Y.M. Vega, J.J.L. Gonzalez, and J.C. Antoranz, Phys. Rev. Lett. 76, 42 (1996).

[4] C. Lewenkopf, J. Dreute, A.A. Magd, J. Aichelin, W. Heinrich, J. Hüfner, G. Rusch, and B. Wiegel, Phys. Rev. C 44, 1065 (1991).
[5] B. Farizon, M. Farizon, M.J. Gaillard, F. Gobet, M. Carré, J.P. Buchet, P. Scheier, and T.D. Mark, Phys. Rev. Lett. 81, 4108 (1998).

[6] Statistical Models for the Fracture of Disordered Media, edited by H.J. Herrmann and S. Roux (Elsevier, Amsterdam, 1990); High-Pressure Shock Compression of Solids II, Dynamic Fracture and Fragmentation, edited by L. Davison, D.E. Grady, and M. Shahinpoor (Springer, Berlin, 1996). 
[7] D.E. Grady and M.E. Kipp, J. Appl. Phys. 58, 1210 (1985).

[8] A. Meibom and I. Balslev, Phys. Rev. Lett. 76, 2492 (1996).

[9] M. Marsili and Y.-C. Zang, Phys. Rev. Lett. 77, 3577 (1996).

[10] M. Sahimi and T.T. Tsotsis, Phys. Rev. Lett. 59, 888 (1987); Chem. Eng. Sci. 43, 113 (1988).

[11] M. Sahimi, Phys. Rev. A 43, 5367 (1991).

[12] K.R. Coutinho, S.K. Adhikari, and M.A.F. Gomes, J. Appl. Phys. 74, 7577 (1993); K.R. Coutinho, M.A.F. Gomes, and S.K. Adhikari, Europhys. Lett. 18, 119 (1992).

[13] G. Gamow, Z. Phys. 51, 204 (1928).

[14] R.W. Gurney and E.U. Condon, Nature (London) 122, 439 (1928).

[15] A. S. Davydov, Quantum Mechanics (Addison-Wesley, New York, 1968).

[16] J. Hoshen and R. Kopelman, Phys. Rev. B 15, 5046 (1976).

[17] D. Stauffer, Introduction to Percolation Theory (Taylor and Francis, London, 1985).

[18] G. Marsaglia, Comput. Math. Appl. 9, 1 (1993).

[19] J.E. Finn, S. Agarwal, A. Bujak, J. Chuang, L.J. Gutay, A.S. Hirsch, R.W. Minich, N.T. Porile, R.P. Scharenberg, B.C. Stringfellow, and F. Turkot, Phys. Rev. Lett. 49, 1321 (1982).

[20] D.L. Turcotte, J. Geophys. Res. 91, 1921 (1986).

[21] L. Oddershede, P. Dimon, and J. Bohr, Phys. Rev. Lett. 71, 3107 (1993).

[22] V.P. Brito, M.A.F. Gomes, F.A.O. Souza, and S.K. Adhikari, Physica A 259, 227 (1998).

[23] D.W. Thompson, On Growth and Form (Cambridge University Press, Cambridge, 1971).
[24] D.M. Raup, S.J. Gould, T.J.M. Schopf, and D.S. Simberloff, J. Geol. 81, 525 (1973).

[25] S. Wolfram, Theory and Applications of Cellular Automata (World Scientific, Singapore, 1986).

[26] B. B. Mandelbrot, The Fractal Geometry of Nature (Freeman, New York, 1983).

[27] U. Frisch, Turbulence (Cambridge University Press, Cambridge, 1996).

[28] G. Parisi, Phys. Scr. 35, 123 (1987).

[29] M.A.F. Gomes, F.A.O. de Souza, and S.K. Adhikari, J. Phys. A 28, L613 (1995).

[30] T. Kadono, Phys. Rev. Lett. 78, 1444 (1997).

[31] L. Balázs, L. Nyikos, I. Szabó, and R. Schiller, Fractals 1, 416 (1993).

[32] G. Wagner, A. Birovljev, P. Meakin, J. Feder, and T. Jøssang, Phys. Rev. E 55, 7015 (1997).

[33] See, e.g., G. Paladin and A. Vulpiani, Phys. Rep. 156, 147 (1987).

[34] A. Coniglio and M. Zannetti, Physica A 163, 325 (1990).

[35] M.A.F. Gomes and T.R.M. Sales, Phys. Lett. A 177, 215 (1993).

[36] K.R. Coutinho, M.D. Coutinho-Filho, M.A.F. Gomes, and A.M. Nemirovsky, Phys. Rev. Lett. 72, 3745 (1994).

[37] C.D. Lorenz and R.M. Ziff, Phys. Rev. E 57, 230 (1998).

[38] A.S. Hirsch, A. Bujak, J.E. Finn, L.J. Gutay, R.W. Minich, N.T. Porile, R.P. Scharenberg, B.C. Stringfellow, and F. Turkot, Phys. Rev. C 29, 508 (1984).

[39] C.B. Chitwood, D.J. Fields, C.K. Gelbke, W.G. Lynch, A.D. Panagiotou, M.B. Tsang, H. Utsunomiya, and W.A. Friedman, Phys. Lett. B 131, 289 (1983). 